\title{
High resolution time of arrival estimation for a cooperative sensor system
}

\author{
C. Morhart and E. M. Biebl \\ Fachgebiet Höchstfrequenztechnik, Technische Universität München, Munich, Germany
}

\begin{abstract}
Distance resolution of cooperative sensors is limited by the signal bandwidth. For the transmission mainly lower frequency bands are used which are more narrowband than classical radar frequencies. To compensate this resolution problem the combination of a pseudo-noise coded pulse compression system with superresolution time of arrival estimation is proposed. Coded pulsecompression allows secure and fast distance measurement in multi-user scenarios which can easily be adapted for data transmission purposes (Morhart and Biebl, 2009). Due to the lack of available signal bandwidth the measurement accuracy degrades especially in multipath scenarios. Superresolution time of arrival algorithms can improve this behaviour by estimating the channel impulse response out of a band-limited channel view. For the given test system the implementation of a MUSIC algorithm permitted a two times better distance resolution as the standard pulse compression.
\end{abstract}

\section{Introduction}

For security systems and a number of logistic applications the combination of clear identification and exact localization is a vital task. Actions and reactions can only be taken if the classification of the target is assured. Cooperative sensors are therefore a key technology as the use of active target transponders offers an easy identification by the transponder signal. Furthermore, the communication between cooperative sensors can even be established in difficult communication scenarios when there's no line of sight. This property is dependent on the transmission frequency and it was shown (Fackelmeier et al., 2008) that lower frequencies are advantageous for the detection of visually hidden objects. Nevertheless, bandwidth is limited for lower frequencies like ISM bands, which are additionaly used by other applications like data transmission. In Morhart and Biebl (2009) a distance measurement system was presented for the protection of vulnerable road users in city scenarios. With this approach in the $2.4 \mathrm{GHz}$ band highly accurate results for line of sight scenarios could be achieved. On the other side, this system offered a path resolution of only $5 \mathrm{~m}$ unidirectionally and respectively $2.5 \mathrm{~m}$ bidirectionally. For the time of arrival (ToA) estimation, the fastest path is also the most important one. Thus, in multipath scenarios with limited resolution a distance error can result because of unresolvable signal arrivals. This is especially disadvantageous in non line of sight scenarios where several comparable strong transmission links occur. A possibility to improve this bandwidth problem is with superresolution ToA estimation (Krim and Viberg, 1996). Here, the complete channel impulse response is predicted out of a bandlimited channel view by superimposing a specific channel model. It has been shown in (Manolakis et al., 2000) that especially eigenstructure based methods like MUSIC and ESPRIT show superior performance. In combination with biphase modulated pulse compression the approach of $(\mathrm{Du}-$ mont et al., 1994) is advantageous. Therein a MUSIC implementation for a CDMA based system is described. This method could easily be adapted for the cooperative distance measurement system from Morhart and Biebl (2009).

\section{System model}

The base system for the implementation of the MUSIC superresolution algorithm is the pedestrian protection system of Morhart and Biebl (2009). This approach uses a Round Trip Time of Flight principle in combination with bi-phase modulated pulse compression for improved measurement accuracy. For a better understanding of this method it is reasonable to repeat the main principles.

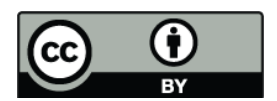

Correspondence to: C. Morhart

(morhart@tum.de)

Published by Copernicus Publications on behalf of the URSI Landesausschuss in der Bundesrepublik Deutschland e.V. 


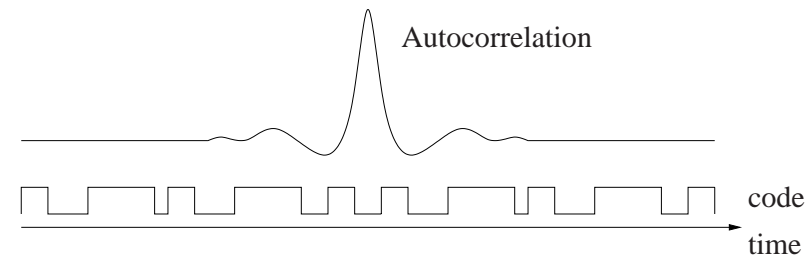

Fig. 1. Schematic capture of biphase modulated pulse compression.

\subsection{Round trip time of flight}

The round trip time of flight (RToF) method resembles the classical radar principle. A signal transmitted by the car is received at the pedestrian side and sent back after a finite waiting time $T_{\mathrm{w}}$. By knowing the waiting time the car can compute the distance $\Delta s$ out of the time $T_{\mathrm{p}}$ passed since the start of transmission.

$\Delta s=\frac{\Delta T}{2} c_{0}=\frac{T_{\mathrm{p}}-T_{\mathrm{w}}}{2} c_{0}$

The advantage of this method is that no absolute time synchonization is needed but only a relative one to determine the exact delay time.

\subsection{Pulse compression}

High precision distance measurement and insensitivity to noise require a high Signal to Noise Ratio (SNR). With limited output power a possible way to increase this ratio is by introducing pulse compression. This method raises the pulse energy by spreading signal information in time domain at constant amplitude and bandwidth (Skolnik, 2001). The energy-gain is due to a larger time bandwidth product that results equivalently in a slower measurement process compared to a single pulse system. An additional advantage of pseudo noise coded pulse compression is a differentiation of multi-user by applying different transmit codes. The ToA is then determined by correlating the received signal with the appropriate code (cf. Fig. 1). In that way the energy of the code sequence is compressed onto the autocorrelation peak, leading to a SNR gain in the ratio of the compression factor.

\subsection{Multipath resolution}

A key property of cooperative sensor technology is that target interference can be avoided by using signal multiplexing techniques like time division multiple access or frequency division multiple access. Nevertheless, each signal of an active transponder can still generate multipath interference due to reflections and diffractions on obstacles in the transmission path. The superposition of these signals leads to different correlation maxima in a pulse compression. The minimum distance between two signals which can still be distinguished is called multipath resolution. In the classical approach the ToA is defined by the correlation peak. Thus, the width of

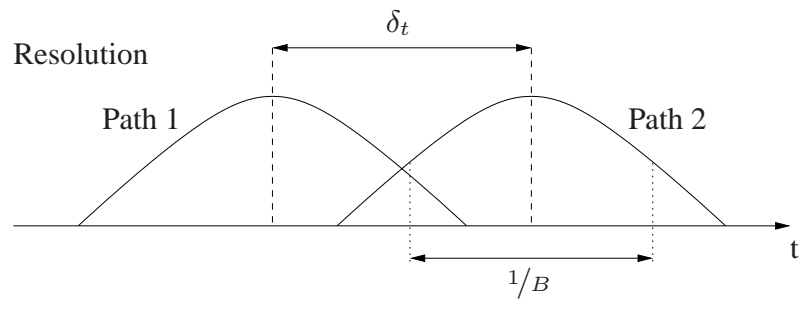

Fig. 2. Resolution between 2 multipath with different time delay.

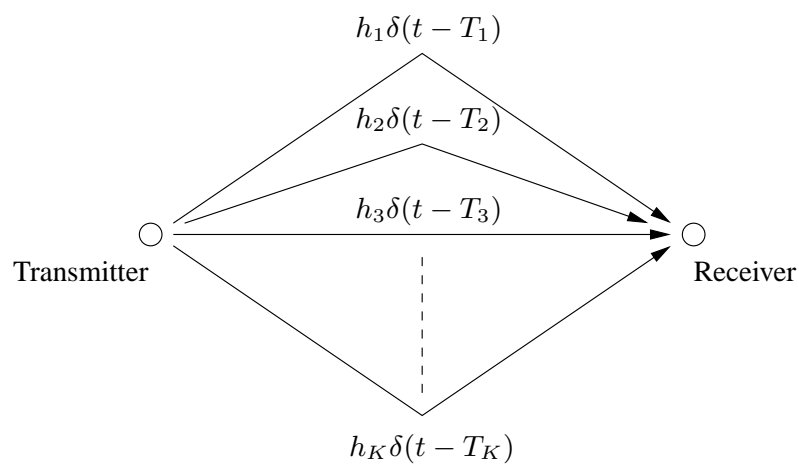

Fig. 3. Channel model.

correlation maximum is proportional to the resolution (cf. Fig. 2). For the case of pulse modulated signal transmission, this resolution can be estimated by the pulse bandwidth (Haykin, 1995):

$\delta_{\mathrm{t}}=\frac{1}{B}$

As signal bandwidth is limited for lower frequency bands, classical multipath resolution is comparatively large.

\subsection{Channel model}

For signal transmission in city scenarios, multipath propagation because of reflection and diffraction on obstacles has to be regarded (cf. Fig. 3). In dependency on the surroundings there are $K$ multipaths having different time delay $T_{k} \in \mathbb{R}_{0}^{+}$ and different path attenuation $h_{k} \in \mathbb{C}$. Therefore, the received signal consists of $K$ replicas of the same signal. Accordingly, the continuous channel impulse response is defined by:

$h(t)=\sum_{k=0}^{K} h_{k} \delta\left(t-T_{k}\right)$

The complex channel impulse factor $h_{k}$ incorporates the channel attenuation and the channel phase variation, which can be assumed randomly distributed between $[0,2 \pi]$.

This channel transfer function can be described as a sum of complex exponentials in frequency domain:

$H(f)=\sum_{k=0}^{K}\left|h_{k}\right| \mathrm{e}^{-j\left(2 \pi f T_{k}+L h_{k}\right)}$ 
Different path delays $T_{k}$ correspond to different oscillation periods, whereas the oscillation phase is randomly distributed. With this channel model a time domain peak search can be changed into the determination of the oscillation period in frequency domain. For this task several superresolution algorithms are available (Krim and Viberg, 1996). For the pedestrian protection system the MUSIC algorithm was chosen because of its superior performance even with low SNR.

\subsection{MUSIC Algorithm}

The MUSIC Algorithm is based on a signal model consisting of a sum of harmonic signals and noise (Schmidt, 1986).

$x[n]=\sum_{k=0}^{K} \alpha_{k} \mathrm{e}^{j \omega_{k} n}+\eta[n]$

Each harmonic function is characterized by a complex amplitude $\alpha_{k} \in \mathbb{C}$ and an oscillation frequency $\omega_{k} \in \mathbb{R} . \quad \eta[n]$ corresponds to the additional noise. This relation can be expressed in matrix-vector notation as:

$\boldsymbol{x}=\sum_{k=0}^{K} \alpha_{k} \boldsymbol{v}\left(\omega_{k}\right)+\boldsymbol{\eta}$

$\boldsymbol{x}=[x[0], x[1], \ldots, x[N-1]]^{T}$ is the $N \times 1$ data vector, $\boldsymbol{v}(\omega)=\left[1, \mathrm{e}^{j \omega}, \ldots, \mathrm{e}^{j(N-1) \omega}\right]^{T}$ the $N \times 1$ complex phase vector and $\eta$ the noise vector. By building the autocorrelation matrix $\mathbf{R}_{x x}$ the correlation result can be divided into the information part $\mathbf{R}_{s s}$ and the noise $\mathbf{R}_{\eta}$.

$$
\begin{aligned}
\mathbf{R}_{x x} & =\mathbf{R}_{s s}+\mathbf{R}_{\eta} \\
\mathbf{R}_{s s} & =\sum_{k=0}^{K}\left|h_{k}\right|^{2} \boldsymbol{v}\left(\omega_{k}\right) \boldsymbol{v}^{H}\left(\omega_{k}\right)
\end{aligned}
$$

The matrix dimension for $\mathbf{R}_{x x}$ is $P \times P$ with $P \leq N . P=N$ means maximum resolution, whereas $P \ll N$ means more redundancy and less computational costs. The noise correlation matrix in the case of white noise is a diagonal-matrix with

$\mathbf{R}_{\eta}=\sigma_{\eta}^{2} \mathbf{E}$.

In the case of $K<P$, The eigenvalue decomposition results in $K$ signal and noise eigenvalues and $P-K$ pure noise eigenvalues.

$\lambda_{p}=P\left|h_{p}\right|^{2}+\sigma_{\eta}^{2} \quad$ for $\quad p \leq K$

$\lambda_{p}=\sigma_{\eta}^{2} \quad$ for $\quad K<p \leq P$

According to these eigenvalues there are $P$ signal- and $P-K$ noise-eigenvectors.

$\mathbf{Q}_{s}=\left[\boldsymbol{q}_{1}, \boldsymbol{q}_{2}, \ldots, \boldsymbol{q}_{K}\right]^{T}$

$\mathbf{Q}_{\eta}=\left[\boldsymbol{q}_{K+1}, \ldots, \boldsymbol{q}_{P}\right]^{T}$

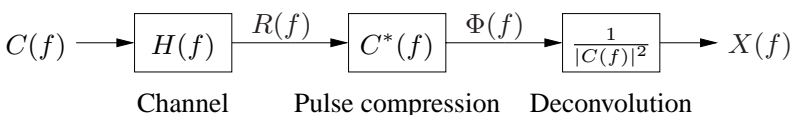

Fig. 4. Idealized schematic of signal processing chain.

Because of the conjugate transposed structure of the correlation matrix all eigenvectors are orthogonal, i.e. $\mathbf{Q}_{s} \perp \mathbf{Q}_{\eta}$. Therefore signal information $h_{k} \boldsymbol{v}\left(\omega_{k}\right)$ is a linear combination of $\mathbf{Q}_{s}$ and orthogonal to $\mathbf{Q}_{\eta}$. This property can be used to determine the signal frequencies $\omega_{k}$. The magnitude of the scalar product with a noise eigenvector $\left|\boldsymbol{v}(\omega) \cdot \boldsymbol{q}_{\eta}\right|$ is minimum if $\omega=\omega_{k}$ and $\boldsymbol{v}\left(\omega_{k}\right)$ a signal vector. Thus, the MUSIC pseudospectrum can be defined as the reciprocal of the sum of scalar products with all noise eigenvectors.

$$
R(\omega)=\frac{1}{\sum_{p=K+1}^{P}\left|\boldsymbol{v}(\omega) \cdot \boldsymbol{q}_{p}\right|^{2}}
$$

\section{System implementation}

Harmonic functions plus noise are the initial point of the MUSIC algorithm. This model matches with the channel transfer function in frequency domain. Thus, for the implementation of this superresolution algorithm a reconstruction of the channel transfer function is necessary. In a bi-phase modulated pulse compression system this is possible by deconvolving with the autocorrelation function. Figure 4 shows an illustration for a baseband transmission without nonlinearities and model errors. Following, the stages of ToA determination will be explained.

\subsection{Correlation}

Pulse compression or equivalently signal correlation can be carried out in time or frequency domain. In time domain correlation can be achieved with a FIR Filter structure, in frequency domain with a scalar product of frequency vectors.

$$
\begin{aligned}
\varphi[n] & =\sum_{N} c[n] r^{*}[n+N] \\
\Phi[m] & =C[m] R^{*}[m]
\end{aligned}
$$

The latter one is computational cost-saving especially for long code sequences. The SNR of the correlation output is also dependent on the code length $L_{c}$.

$\mathrm{SNR}_{k}=\frac{2\left|h_{k}\right|^{2} L_{c} E_{\mathrm{p}}}{N_{0}}$

$E_{\mathrm{p}}$ is the pulse energy and $N_{0}$ the noise power density. For maximum target dynamic it is important to choose codes with minimum correlation side lobes like $\mathrm{m}$-sequences and Barker codes. 


\subsection{Deconvolution}

For optimum performance of superresolution algorithms a maximum similarity between real communication and the chosen signal model is needed. The pure correlation result is therefore not suited as additional components like correlation pulse form, spectral filter characteristics or hardware nonlinearities are also included. The goal is to cancel those parts out of the received signal. This method is called Deconvolution and carried out by a division with the correlation power density.

$X[m]=\frac{\Phi[m]}{|C[m]|^{2}} \approx H[m]$

With the neglect of model nonidealities, this result matches with the channel impulse response. In a real implementation not only the correlation pulse form but also spectral filter charactersitics should be compensated. In this context, digital filters are advantegeous as the filter coefficients can be exactly known on transmitter and receiver side.

\subsection{MUSIC implementation}

For the computation of the MUSIC pseudospectrum the parameter $P$ and $K$ have to be determined. $P$ describes the dimension of $\mathbf{R}_{x x}$ and is therefore a degree for the frequency aperture of the algorithm (Manolakis et al., 2000). Thus larger values of $P$ have better resolution capabilities whereas smaller values result in more signal averaging and less variations in the determination of noise eigenvalues. Another convenient characteristic of smaller values of $P$ is a decreasing computational effort. In the literature there's no optimal solution for the relation of $P$ to $N$ with values ranging from $P \in[N / 3,3 N / 5]$ (Li and Pahlavan, 2004). With regard to signal processing costs for mobile transponder $P=N / 3$ was chosen.

Another important point is the estimation of the included signal components $K$. This can be done with the result of the eigenvalue decomposition. In the case of an adequate SNR ratio, signal eigenvalues outrange noise eigenvalues. Hence, there's a detectable step between signal and noise subspace. For the following section, especially the case $K=2$ was examined.

\section{Verification}

The benefit of superresolution algorithms for cooperative sensor technology lies in an increase of ToA resolution. This improvement is possible because of the reconstruction of a limited channel view with an assumed signal model. Nevertheless the detected channel information and thus the signal bandwidth has still a deciding influence on the quality of this method.

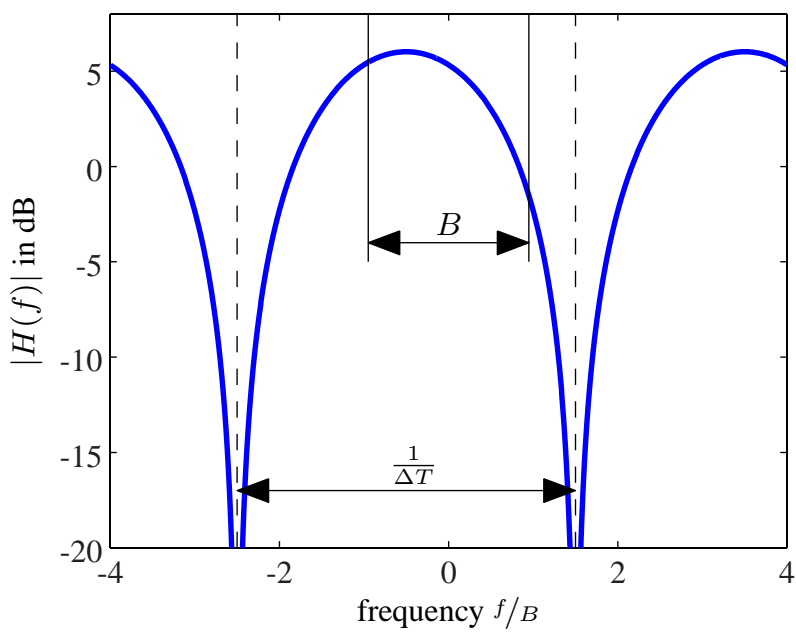

Fig. 5. Magnitude of $|H(f)|$ for time difference $\Delta T=1 / 4 \delta_{t}$ and $\Delta \varphi=-\pi / 4$.

\subsection{Channel information}

At first the available channel information should be analyzed. For superresolution ToA determination only scenarios with at least two transmission paths and time difference $\Delta T$ smaller than the classical resolution $\delta_{t}$ are interesting. Figure 5 shows the spectral magnitude of a channel transfer function for the case of a two path transmission with a time difference of $\Delta T=1 / 4 \delta_{t}$ and a phase difference of $\Delta \varphi=-\pi / 4$. The detectable channel spectrum is limited by the transmission bandwidth $B$, so that only a small section of the channel information is visible. This section is furthermore dependent on the phase difference which can be assumed randomly distributed between $\Delta \varphi \in[0,2 \pi]$. As a consequence the superresolution results are connected to the position of the channel extract. If concentrated at a fading point, the SNR and thus the quality of channel reconstruction is degrading. Hence, the influence of the SNR on the MUSIC resolution was investigated.

\subsection{Simulation of multipath resolution}

For the determination of the resolution boundary a simulation with a two path transmission with variable time difference and SNR was executed. Transmission parameters were chosen according to the proposed cooperative sensor system from Morhart and Biebl (2009) with 256 Bit long $\mathrm{m}$-sequences and a two times oversampling receiver. The phase difference was assumed randomly distributed between $[0,2 \pi]$. Figure 6 shows the resolution boundary for the MUSIC and the correlation ToA determination. The correlation boundary is according to Eq. (2) at $\Delta T=\delta_{t}$ with two resolved paths for $\Delta T>\delta_{t}$.

The situation is different for the MUSIC algorithm. Simulation results for the resolution boundary show a relation 


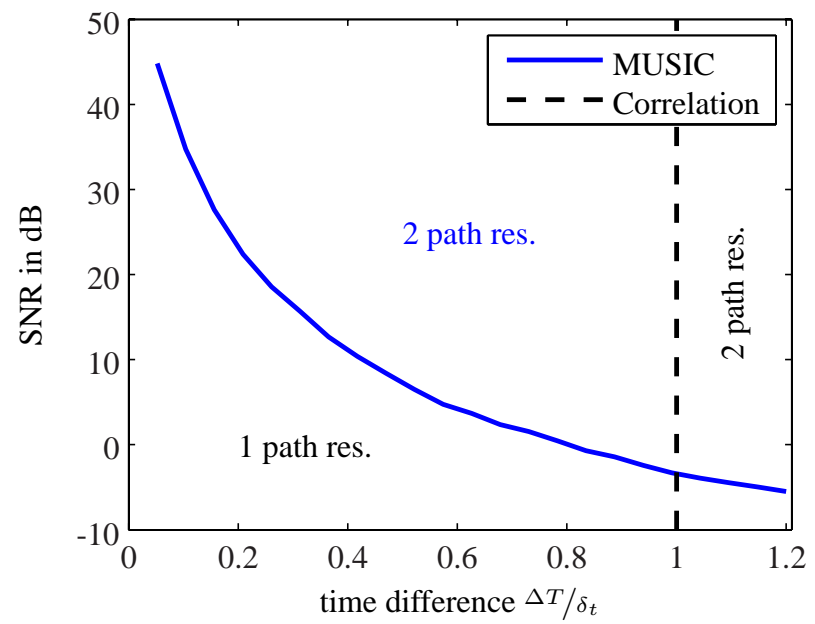

Fig. 6. Simulation of resolution boundary for two path transmission with variable time difference $\Delta T$ and SNR.

$$
\Delta T=(0.2-2) \delta_{t}
$$

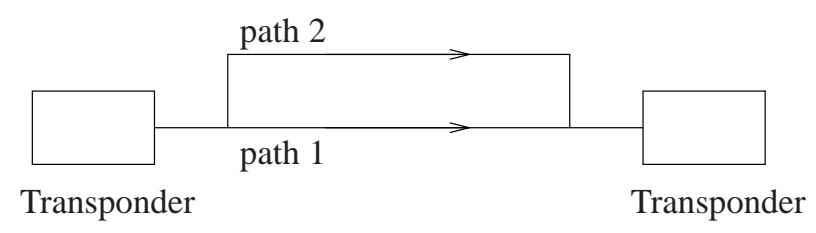

Fig. 7. Measurement assembly for the determination of resolution boundary.

between the time difference and the SNR. Summarized, 2 paths can even be resolved below the classical resolution boundary if the SNR is sufficient. Unfortunately, for too small time differences huge SNR values are necessary, what makes a resolution unlikely. The big advantage for a pulse compression system is that ToA resolution together with the SNR can be adapted with the length of the code sequence.

\subsection{Test measurement}

To check out the performance of the MUSIC algorithm for a cooperative sensor system test measurements were done with a prototype hardware. Two transponders were used which were connected via coaxial cable (cf. Fig. 7). The signal was divided into two transmission paths with variable time difference $\Delta T$. This factor was modified by changing the cable length difference. Furthermore, to equal amplitude levels additional attenuation elements were used into the shorter path. On the receiver side the correlation result was passed onto a PC to compute the superresolution method. This is necessary because the MUSIC algorithm was not implemented on the receiver FPGA yet. In Fig. 8 the measured time difference between the two paths is illustrated. If they can not be distinguished the difference drops to zero. It can be seen, that MUSIC post processing offers a more than two times bet-

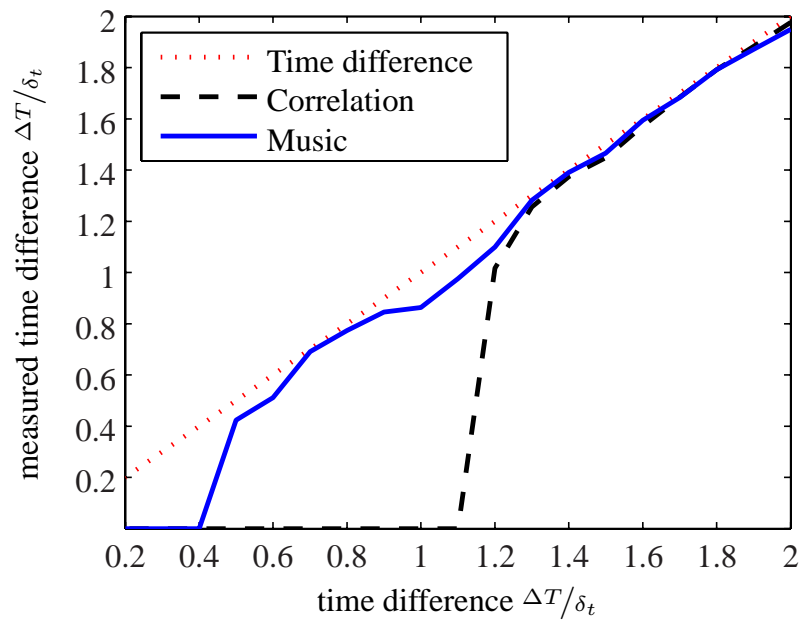

Fig. 8. Measurement of signal resolution for two path transmission with variable time difference.

ter path resolution compared with the correlation result. It is also remarkable that correlation does not reach the theoretical resolution limit. This is due to pulse widening in the transmission process. Another problem of the actual prototype are nonidealities and model errors because of uncompensated filter and amplifier charateristics. Even though superresolution post processing is able to significantly improve the ToA resolution.

\section{Conclusions}

Cooperative sensor systems have to use the same signal bands as data communication systems. Bandwidth in these bands is tightly limited resulting equivalently in a low path resolution. The application of superresolution methods is therefore a suitable alternative for the improvement of ToA determination. In the simulation it could be shown that the resolution of the MUSIC algorithm is limited by the SNR and the time difference. For the proof of this fact a MUSIC ToA algorithm was implemented into a existing cooperative sensor system. Measurement results demonstrated a more than two times higher path resolution compared with the standard pulse compression although a non ideal sensor hardware has been used. The next steps are therefore a minimization of model errors by improving hardware linearity and an implementation of the MUSIC algorithm on the transponder FPGA. 


\section{References}

Dumont, L., Fattouche, M., and Morrison, G.: Super-resolution of multipath channels in a spread spectrum location system, Electronics Lett., 30, 1583-1584, 1994.

Fackelmeier, A., Morhart, C., and Biebl, E.: Evaluation of Diffraction Effects for Identifying Hidden Targets, in: GeMIC 2008 ITG-Fachbericht Band 206, 2008.

Haykin, S.: Adaptive Filter Theory, Prentice Hall, 1995.

Krim, H. and Viberg, M.: Two decades of array signal processing research: the parametric approach, Signal Processing Magazine, IEEE, 13, 67-94, 1996.

Li, X. and Pahlavan, K.: Super-resolution TOA estimation with diversity for indoor geolocation, Wireless Communications, IEEE Transactions on, 3, 224-234, 2004.
Manolakis, D. G., Ingle, V. K., and Kogon, S. M.: Statistical and Adaptive Signal Processing: Spectral Estimation, Signal Modeling, Adaptive Filtering, and Array Processing, McGraw Hill, New York, 2000.

Morhart, C. and Biebl, E. M.: Cooperative multi-user detection and ranging based on pseudo-random codes, Adv. Radio Sci., 7, 5559, 2009, http://www.adv-radio-sci.net/7/55/2009/.

Schmidt, R.: Multiple emitter location and signal parameter estimation, Antennas and Propagation, IEEE Transactions on, 34, 276-280, 1986.

Skolnik, M. I.: Introduction to RADAR systems, chap. 6, pp. 317331, McGraw-Hill, New York, 3. edn., 2001. 